\title{
A randomised, prospective study comparing selective laser trabeculoplasty with latanoprost for the control of intraocular pressure in ocular hypertension and open angle glaucoma
}

\author{
M Nagar, A Ogunyomade, D P S O'Brart, F Howes, J Marshall
}

\begin{abstract}
Aim: To compare $90^{\circ}, 180^{\circ}$, and $360^{\circ}$ selective laser trabeculoplasty (SLT, $532 \mathrm{~nm} \mathrm{Nd:YAG} \mathrm{laser)} \mathrm{with} \mathrm{latano-}$ prost $0.005 \%$ for the control of intraocular pressure (IOP) in ocular hypertension (OHT) and open angle glaucoma (OAG).

Methods: A prospective, randomised clinical trial in the Department of Ophthalmology, St Thomas's Hospital, London, and Clayton Eye Centre, Wakefield, West Yorkshire. 167 patients (167 eyes) with either OHT or OAG were randomised to receive $90^{\circ}, 180^{\circ}$, and $360^{\circ} \mathrm{SLT}$ or latanoprost $0.005 \%$ at night and were evaluated at 1 hour, 1 day, 1 week and 1, 3, 6, and 12 months.

Results: The mean follow up was 10.3 months (range 112 months). Early, transient, complications such as postoperative ocular pain, uveitis, and 1 hour IOP spike occurred in a number of eyes after SLT, with pain being reported more frequently after $360^{\circ}$ than $90^{\circ}$ treatments $(p>0.001)$. Success rates defined in terms of both a $20 \%$ or more and a $30 \%$ or more IOP reduction from baseline measurements with no additional antiglaucomatous interventions were better with latanoprost than $90^{\circ}(p<0.001)$ and $180^{\circ}$ SLT $(p<0.02)$ treatments. Differences in success rates between latanoprost and $360^{\circ}$ SLT did not reach statistical significance $(p<0.5)$. Success rates were greater with $180^{\circ}$ and $360^{\circ}$ compared to $90^{\circ} \mathrm{SLT}(\mathrm{p}<0.05)$. With $360^{\circ} \mathrm{SLT}, 82 \%$ of eyes achieved a $>20 \%$ IOP reduction and $59 \%$ a $>30 \%$ reduction from baseline. Although success rates were better with $360^{\circ}$ than $180^{\circ}$ SLT treatments, differences did not reach statistical significance. There were no differences with regard to age, sex, race, pretreatment IOP, OHT versus OAG, laser power settings, and total laser energy delivered between eyes which responded, in terms of $a>20 \%$ and $a>30 \%$ IOP reduction, and those that did not respond with $180^{\circ}$ and $360^{\circ}$ SLT treatments.

Conclusions: Success rates were higher with latanoprost $0.005 \%$ at night than with $90^{\circ}$ and $180^{\circ} \mathrm{SLT}$ treatments. $90^{\circ}$ SLT is generally not effective. $360^{\circ}$ SLT appears to be an effective treatment with approximately $60 \%$ of eyes achieving an IOP reduction of $30 \%$ or more. Transient anterior uveitis with associated ocular discomfort is not unusual in the first few days after SLT. Late complications causing ocular morbidity after SLT were not encountered.
\end{abstract}

$\mathrm{T}$ he technique of argon laser trabeculoplasty (ALT) was first reported in 1979 by Wise and Witter ${ }^{1}$ and has since gained acceptance as a viable treatment option for open angle glaucoma (OAG). ${ }^{2}$ The Glaucoma Laser Trial Research group have indicated that eyes initially treated with ALT tend to have lower intraocular pressures (IOP) and better visual field and optic disc status than fellow eyes receiving medical treatment. ${ }^{2}$ The precise mechanism of ALT, however, remains unclear. Response to treatment varies and late failure is not uncommon. Histological studies of ALT burns indicate destruction of the uveoscleral meshwork with surrounding thermal damage, together with membrane formation over the meshwork by migrating endothelial cells, ${ }^{3-5}$ which it has been postulated, may be responsible for long term loss of effect.

Recently a new approach to laser trabeculoplasty has been introduced with the development of selective laser trabeculoplasty (SLT), in which a Q switched, frequency doubled, neodymium:yittrium-aluminium-garnet (Nd:YAG) laser emitting at $532 \mathrm{~nm}$ is used to selectively target pigmented trabecular cells without causing thermal damage to adjacent non-pigmented meshwork structures. ${ }^{6}$ Clinical trials have been encouraging, with reasonable response rates, moderately effective IOP reduction and minimal side effects. ${ }^{7-11}$ Comparable studies have shown SLT to be as effective as ALT, ${ }^{12}{ }^{13}$ while histological investigations have demonstrated less damage to the ultrastructure of the trabecular meshwork. ${ }^{14} 15$

As a result of these studies, SLT has been advocated as a useful treatment in the management of OAG with a role as a possible primary treatment. However, its mechanism of action is unclear. Success rates and levels of reported IOP reduction vary between studies and optimal energy settings and responses to the degree of angle treated as yet remain undetermined. In order to address some of these issues we conducted a randomised, prospective study comparing $90^{\circ}$, $180^{\circ}$, and $360^{\circ}$ SLT with latanoprost $0.005 \%$ at night for the control of intraocular pressure in ocular hypertension (OHT) and OAG. The aims of the study were to investigate the potential of SLT as a primary therapeutic modality, to examine responses to the degree of angle treated and elucidate factors in terms of patient demographics, laser settings, and type of glaucoma that could influence the efficacy of SLT.

\section{METHODS}

\section{Study design}

The study was designed (by DO, MN, and JM) as a prospective, randomised, controlled trial and conducted at

Abbreviations: ALT, argon laser trabeculoplasty; IOP, intraocular pressure; OAG, open angle glaucoma; OHT, ocular hypertension; PDS, pigment dispersion syndrome; PXE, pseudoexfoliation syndrome; SLT, selective laser trabeculoplasty 
two treatment centres: the Department of Ophthalmology, St Thomas's Hospital, London, and the Clayton Eye Centre, Wakefield, West Yorkshire. Ethics committee approval was obtained at both sites. Randomisation was performed using a sealed, shuffled envelope system, in which an allocated treatment (latanoprost and $90^{\circ}, 180^{\circ}$, and $360^{\circ}$ of SLT) was written on treatment cards. These cards were placed into identical sealed envelopes, which were then shuffled several times and sequentially numbered. No patient identifiers were used in this process. None of the individuals involved in generating the randomisation took any further part in the study. The sealed envelopes (separate sets for each of the two treatment centres) were kept in locked draws, which were unlocked before treatment, when the next available numbered envelope was opened and the patient allocated to a treatment group according to the modality written on the card. If indicated, both eyes of each patient received identical treatments on the basis of randomisation. However, only one eye of each patient was entered into the study. This was either the eye with the highest IOP measurement at baseline examination or, if the pressures were identical, the right eye was chosen. It was not possible to mask either the treating ophthalmologist $(\mathrm{MN}, \mathrm{AO})$ or the patient as this was a medical versus surgical intervention trial. Because of the apparent lack of efficacy with $90^{\circ}$ SLT treatments, 9 months after commencement of the study randomisation the $90^{\circ}$ group was discontinued at the Clayton Eye Centre but was continued at St Thomas's Hospital.

Of the 193 patients who were assessed for eligibility, 26 were excluded ( 12 did not meet the inclusion criteria, seven did not wish to participate, and nine were unable to comply with the intended follow up commitment).

\section{Subjects}

One hundred and sixty seven patients (167 eyes) with OHT (85 eyes) or OAG (82 eyes) were randomised, between January 2002 and January 2003, to receive either latanoprost $0.005 \%$ at night ( 39 eyes) or $90^{\circ}$ ( 35 eyes), $180^{\circ}$ ( 49 eyes), or $360^{\circ}$ (44 eyes) SLT treatments. Patients were recruited from the glaucoma services at the Department of Ophthalmology, St Thomas's Hospital, London and the Clayton Eye Centre, Wakefield. Sixty nine patients (69 eyes) were recruited from St Thomas's and 98 patients (98 eyes) from the Clayton Eye Centre. Before entry into the study, informed consent was obtained from all patients. Inclusion criteria for the study were OHT or primary or secondary OAG, either newly diagnosed or controlled on medical therapy. Exclusion criteria were congenital glaucoma, any type of angle closure glaucoma, eyes with previous laser or surgical glaucoma interventions, and eyes with previous anterior segment surgery. Patients were not excluded from the study on the basis of their age, race, and number and types of antiglaucomatous medications.

Before treatment all patients who had been receiving antiglaucomatous medications underwent a minimum of a 5 week "washout" period. Baseline data were obtained for each patient before initiation of treatment, which included a full ocular and medical history, visual field assessment (Humphrey 24-2 computerised perimetry), slit lamp biomicroscopy, Goldmann applanation tonometry, gonioscopy, and mydriatic funduscopy.

\section{Laser techniques}

Immediately before the laser procedure a single application of amethocaine $1 \%$ was instilled into the operative eye. Two surgeons ( $\mathrm{MN}$ and $\mathrm{AO}$ ) performed all laser treatments.

A Coherent Selectra 7000 laser (Lumenis, Coherent, Inc, Palo Alto, CA, USA) was used in all cases. This is a frequency doubled, q-switched Nd:YAG laser emitting at $532 \mathrm{~nm}$, with a pulse duration of $3 \mathrm{~ns}$, a spot size of $400 \mu \mathrm{m}$, and pulse energies ranging from $0.2-1.7 \mathrm{~mJ}$, coupled to a slit lamp delivery system with a helium-neon laser (HeNe) aiming system.

With the patient seated at the slit lamp system, a Latina single mirror goniolens was placed on the eye. The laser was focused on the trabecular meshwork using the HeNe aiming beams. With the $400 \mu \mathrm{m}$ spot size the entire width of the trabecular meshwork was irradiated with each pulse. The laser energy was initially set at $0.8 \mathrm{~mJ}$ and a single laser pulse was delivered at the $12 \mathrm{o}^{\prime}$ clock position. If a cavitation bubble appeared the laser energy was reduced by $0.1 \mathrm{~mJ}$ increments until no bubble formation was observed and treatment was continued at this energy level. If no cavitation bubble was observed, the pulse energy was increased by increments of $0.1 \mathrm{~mJ}$ until bubble formation and then decreased as described above. During laser application, bubble formation was monitored with each pulse and if a bubble appeared, the pulse energy was decreased as described above.

In patients receiving $90^{\circ}$ treatments 25-30 non-overlapping laser spots were applied to 3 clock hours of the inferonasal or inferotemporal trabecular meshwork. For $180^{\circ}$ treatments 48-53 spots were applied over the inferior 6 clock hours and with $360^{\circ}$ procedures the entire meshwork was treated with 93-102 non-overlapping spots. The total number of pulses delivered and the total amount of energy delivered were recorded following each treatment.

\section{Postoperative management}

Postoperatively, patients were prescribed either dexamethasone $0.1 \%$ eye drops four times a day for 5 days (AO) or ketorolac eye drops four times a day for 5 days $(\mathrm{MN})$.

Patients were examined (by $\mathrm{MN}, \mathrm{AO}$, and $\mathrm{DO}$ ) at 1 hour, 1 day and 1, 3, 6, 9, and 12 months. At each visit patients were invited to report any symptoms of ocular morbidity and an ophthalmic examination was performed, which included visual acuity measurement, slit lamp biomicroscopy, and Goldmann applanation tonometry. The observers were not masked to treatment arm. In addition, visual field assessment, gonioscopy, and funduscopy were performed at 12 months.

\section{Success criteria}

Success was defined both as a $20 \%$ or more reduction in IOP from baseline measurements and also as a $30 \%$ or greater IOP reduction from baseline with no additional antiglaucomatous interventions. During the follow up period a number of eyes did not achieve adequate IOP control and were deemed unsuccessful. At the discretion of the treating surgeons (AO, $\mathrm{MN}$, and DO) further laser treatments or antiglaucomatous

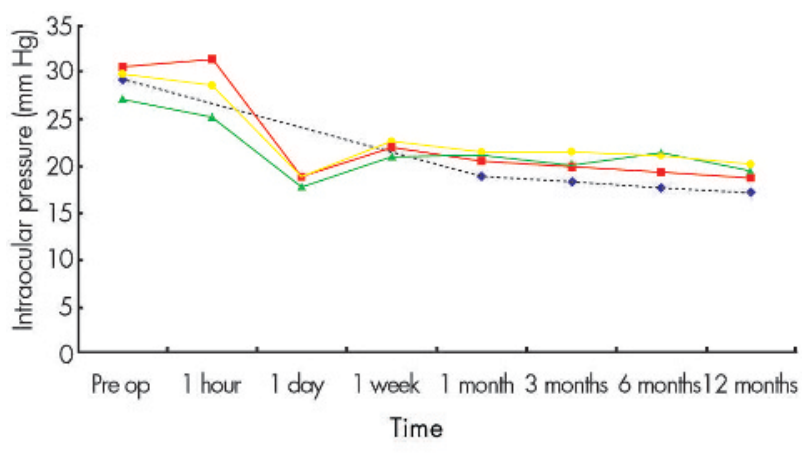

Figure 1 Intraocular pressure over time with treatment with latanoprost (blue diamonds), $90^{\circ} \mathrm{SLT}$ (green triangles), $180^{\circ} \mathrm{SLT}$ (yellow squares), and $360^{\circ} \mathrm{SLT}$ (red squares). 
medications were administered to ensure adequate IOP control.

\section{Statistical methods}

Before submission to the ethics committee, a power statement was calculated by the Department of Medical Statistics, Guy's and St Thomas's NHS Trust. Using the Ancova method and based on 0.9 power to detect a significant difference $(p=0.05$, two sided $)$, and assuming a standard deviation of $4 \mathrm{~mm} \mathrm{Hg}$, it was estimated that 17 eyes were required for each study group.

Student's $t$ tests were used to compare continuous variables between the groups such as IOP differences. $\chi^{2}$ Analysis was used to compare qualitative data. Results with $\mathrm{p}<0.05$ were considered statistically significant.

\section{RESULTS}

\section{Patient demographics}

Mean age was 63 years (range 22-90). Seventy seven patients were male and 90 were female. Thirty six patients $(22 \%)$ were either of African or Afro-Caribbean origin and 131 patients $(78 \%)$ were white. In 82 eyes $(49 \%)$ a diagnosis of OAG had been made, 76 of which were primary (POAG), four of which were secondary to pigment dispersion syndrome (PDS) and two secondary to pseudoexfoliation syndrome (PXE). Eighty five eyes $(51 \%)$ had a diagnosis of OHT.

Thirty nine patients (39 eyes) were randomised to receive latanoprost $0.005 \%$ at night. Thirty five patients (35 eyes) were randomised to the $90^{\circ}$ SLT group, 49 (49 eyes) to $180^{\circ}$ SLT group, and 44 (44 eyes) to the $360^{\circ}$ SLT group. There were no differences between the four treatment groups in terms of age, sex, race, and aetiology of raised IOP.

\section{IOP control}

Mean baseline IOP in the 167 eyes was $29.3 \mathrm{~mm} \mathrm{Hg}$ (range 22-50 mm Hg, median $28 \mathrm{~mm} \mathrm{Hg}$ ). Mean baseline IOP was lower in the $90^{\circ}$ compared to the other treatment groups $(\mathrm{p}>0.05)$, otherwise there were no differences in baseline IOP between those eyes randomised to receive either latanoprost $0.005 \%$ or $180^{\circ}$ or $360^{\circ}$ SLT.

Mean IOP values with time (including IOP measurements in eyes that required additional SLT and antiglaucomatous medications during the follow up period) for the four treatment groups are shown in figure 1. Mean IOP was significantly lower in eyes receiving latanoprost than $90^{\circ}$ SLT at 1,6 , and 12 months $(\mathrm{p}>0.02), 180^{\circ}$ SLT at $1,3,6$, and 12 months $(p>0.01)$, and $360^{\circ}$ SLT at 12 months $(p<0.05)$. There were no differences in mean IOP values between eyes undergoing $180^{\circ}$ SLT and $90^{\circ}$ SLT during the follow up period. Mean IOP was greater at 1 hour after $360^{\circ}$ than $90^{\circ}$ SLT $(\mathrm{p}<0.001)$. It was lower with $360^{\circ}$ SLT at 6 months than $90^{\circ}$ SLT $(\mathrm{p}<0.05)$ and at 3 months and 6 months compared to $180^{\circ}$ SLT $(\mathrm{p}<0.05)$.

The mean follow up in the 167 eyes was 10.3 months (range 1-12 months). In the latanoprost group, 35 eyes $(90 \%)$ achieved a $>20 \%$ IOP reduction and 28 eyes $(78 \%)$ a $>30 \%$ IOP reduction from baseline measurements with no additional antiglaucomatous interventions. These success rates were significantly higher than those achieved with $90^{\circ}$ SLT $(p<0.001)$, where only 12 eyes $(34 \%)$ achieved a $>20 \%$
IOP reduction and four eyes $(11 \%)$ a $>30 \%$ reduction. Similarly, success rates were significantly higher with latanoprost compared to $180^{\circ}$ SLT $(p<0.01)$, where 32 eyes $(65 \%)$ achieved a $>20 \%$ IOP reduction and 21 eyes $(48 \%)$ a $>30 \%$ reduction. With $360^{\circ}$ SLT, 36 eyes $(82 \%)$ achieved a $>20 \%$ IOP reduction and 26 eyes $(59 \%)$ a $>30 \%$ reduction from baseline. Differences in success rates between latanoprost and $360^{\circ}$ SLT did not reach statistical significance $(p<0.5)$, but were greater with $180^{\circ}$ and $360^{\circ}$ than $90^{\circ}$ SLT $(p<0.05)$. Although success rates were better with $360^{\circ}$ than $180^{\circ}$ SLT treatments, these differences did not reach statistical significance $(\mathrm{p}<0.1)$. Treatment failure generally occurred during the early follow up period. Of the 25 eyes that failed to achieve a $>20 \%$ IOP reduction from baseline after $180^{\circ}$ and $360^{\circ}$ SLT treatments, 18 eyes $(72 \%)$ failed within the first 3 months and four eyes after 6 months.

During the follow up period, at the discretion of the treating surgeon further laser treatments or antiglaucomatous medications were administered to a number of eyes to ensure satisfactory IOP reduction. No eyes underwent glaucoma drainage surgery. Typically, these interventions occurred after 3 months follow up. The number of eyes receiving further SLT treatments and/or medications at the last follow up visit (mean 10.3 months) were four (10\%) in the latanoprost group, 23 (66\%) with $90^{\circ}$ SLT treatments, 17 $(35 \%)$ with $180^{\circ}$ SLT and $11(25 \%)$ in the $360^{\circ}$ SLT group. Significantly, fewer eyes required additional glaucoma interventions with latanoprost compared to the $90^{\circ}$ and $180^{\circ}$ SLT groups $(p>0.01)$. Similarly, fewer interventions were required after $360^{\circ}$ compared to $90^{\circ}$ SLT. Differences were not statistically significant between $180^{\circ}$ and $360^{\circ}$ SLT and the latanoprost and $360^{\circ}$ SLT groups.

Comparison of demographic and treatment parameters in eyes that achieved successful outcomes after $180^{\circ}$ and $360^{\circ}$ SLT, both in terms of a $>20 \%$ IOP reduction $(n=68)$ and a $>30 \%$ IOP reduction $(n=47)$ from baseline, with those eyes in which treatment failed, revealed no differences with regard to age, sex, race, pretreatment IOP, OHT versus OAG, laser power settings, and total laser energy delivered. Of the six eyes with OAG secondary to PDS or PXE, four eyes failed to respond to $180^{\circ}$ and $360^{\circ} \mathrm{SLT}$, with one eye responding but failing to reach a target pressure of less than $22 \mathrm{~mm} \mathrm{Hg}$.

\section{Adverse events}

Transient ocular discomfort and mild uveitis were reported and seen during the first week after SLT treatment (table 1). Such events were more common after $180^{\circ}$ and $360^{\circ}$ SLT than $90^{\circ} \mathrm{SLT}$, but with the exception of ocular pain being reported more frequently after $360^{\circ}$ compared to $90^{\circ}$ treatments $(p>0.001)$ the differences were not statistically significant After $360^{\circ}$ SLT, transient ocular pain was reported in 17 eyes $(39 \%)$ and transient uveitis in $22(50 \%)$. Spikes of IOP at 1 hour ( $5 \mathrm{~mm} \mathrm{Hg}$ or more) were seen in three eyes (9\%) after $90^{\circ}$ SLT, eight eyes (16\%) after $180^{\circ}$ SLT, and 12 eyes $(27 \%)$ after $360^{\circ}$ SLT. Mean IOP at 1 hour was significantly higher with $360^{\circ}$ compared to $90^{\circ}$ SLT treatments $(\mathrm{p}<0.05)$ (fig 1 ).

Sight threatening adverse events did not occur during the follow up period. There were no differences in terms of mean Snellen equivalent best corrected visual acuities, measured cup/disc ratios and average values of mean defects (decibels)

Table 1 Percentage of eyes with transient adverse events reported during the first week after treatment

\begin{tabular}{lllll}
\hline Adverse event & Latanoprost & $\mathbf{9 0 ^ { \circ }}$ SLT & $\mathbf{1 8 0 ^ { \circ } \text { SLT }}$ & $\mathbf{3 6 0 ^ { \circ } \text { SLT }}$ \\
\hline Discomfort/pain & $0 \%$ & $6 \%$ & $20 \%$ & $39 \%$ \\
Uveitis & $0 \%$ & $31 \%$ & $41 \%$ & $50 \%$ \\
IOP spike & $0 \%$ & $11 \%$ & $16 \%$ & $27 \%$ \\
\hline
\end{tabular}


on Humphrey 24-2 visual field testing between baseline measurements and those measured in eyes reaching 12 month follow up in any of the four treatment groups.

\section{DISCUSSION}

This randomised, prospective study was undertaken to investigate the potential of SLT as a primary therapeutic modality for the control of IOP in OHT and OAG, to investigate responses to the degree of angle treated and factors in terms of patient demographics, laser settings, and aetiological issues that could influence the response to SLT treatment. Previous studies investigating the efficacy of ALT as a primary treatment for POAG compared to medical therapy have indicated favourable results. ${ }^{216}$ However, these studies were instigated before the introduction of prostaglandin analogues, which with their efficacy of IOP reduction and excellent safety profile, are now the mainstay of medical antiglaucomatous therapy. For any treatment to be considered as a primary therapy in glaucoma and OHT it must now be compared to a prostaglandin analogue. This current study clearly demonstrated better IOP control in terms of overall success rates with latanoprost compared to either $90^{\circ}$ or $180^{\circ}$ SLT treatments. It was only with $360^{\circ}$ SLT that success rates appeared to approach those with latanoprost suggesting its potential as a primary treatment.

This current study demonstrated a greater IOP lowering response with $360^{\circ}$ and $180^{\circ}$ SLT laser application than $90^{\circ}$ SLT. This is in contrast with a previous study which reported a similar pressure lowering effect with $90^{\circ}$ and $180^{\circ}$ treatments. ${ }^{17}$ The mechanism of action of SLT is not clear. The possible "dose-response" of SLT in terms of the degree of angle treated is interesting. Although differences between $180^{\circ}$ and $360^{\circ}$ SLT did not reach statistical significance, success rates were better with $360^{\circ} \mathrm{SLT}$ and while latanoprost was clearly more efficacious than $180^{\circ}$ SLT differences between latanoprost and $360^{\circ}$ treatments were not statistically significant. While our study demonstrates that treating more than $90^{\circ}$ of the meshwork with SLT is beneficial, there is also likely to be some advantage in terms of successful IOP reduction from treating the whole $360^{\circ}$ of the angle at each laser session rather than just $180^{\circ}$.

Response rates and IOP reduction in this present study were comparable to those reported in the published literature where success rates vary from $64 \%$ to $89 \%$ depending on the definitions used..$^{7-15}$ Comparison of eyes with successful and unsuccessful outcomes after $180^{\circ}$ and $360^{\circ}$ SLT in our study elucidated no differences in terms of age, sex, racial origin, pretreatment IOP, and severity of glaucoma. Although the mechanism of action is unknown, the lack of coagulative damage after SLT, $^{3-6}$ does suggest a possible reaction at a cellular level. It is of interest that while there were no differences in response rates in terms of race and therefore angle pigmentation and also between eyes with either OHT or POAG, the four of the six eyes with PDS and PXE failed to respond. In a previous study in which a group of eyes with capsular (pseudoexfoliation) glaucoma were treated, a trend towards a poorer response was noted. ${ }^{18}$ This is of interest as studies of ALT have suggested better responses in groups of patients with pigmentation of the drainage angle (pigmented races, capsular glaucoma and PDS). ${ }^{19}$ This is not surprising with ALT where coagulative damage is known to occur. However, in SLT where the mechanism may be at a cellular level, debris in the drainage angle in terms of iris pigment and pseudoexfoliation material may prevent adequate laser/ tissue interaction.

It has been postulated that membrane formation over the trabecular meshwork by migrating endothelial cells, ${ }^{3-5}$ may be responsible for long term loss of effect seen after ALT. This long term failure limits the usefulness of the technique. One of the suggested theoretical benefits of SLT in relation to its minimal ultrastructural damage to the trabecular meshwork may be better long term IOP control. Although our follow up is limited, only four eyes $(6 \%)$ after $180^{\circ} / 360^{\circ}$ SLT with an initial successful outcome ( $>20 \%$ IOP reduction from baseline) subsequently "failed." While this is encouraging, further follow up is required to evaluate long term response rates to SLT.

In this study, the overall success rates with $360^{\circ}$ SLT, with $80 \%$ of eyes achieving a $20 \%$ or more IOP reduction and $60 \%$ a $30 \%$ or more IOP reduction, were very encouraging. Long term problems causing ocular morbidity did not occur and early complications were transient and self limiting. Such factors suggest that $360^{\circ}$ SLT may be a useful primary therapeutic modality for the treatment of OHT and OAG. Published studies have demonstrated similar efficacy for ALT and SLT, but with the theoretical advantages of less damage to the ultrastructure of the trabecular meshwork. Long term studies will determine whether such advantages translate into better long term IOP control than with ALT which limits its usefulness. Further investigations are required to determine the precise mechanism of action and tissue interactions of SLT so that strategies to enhance response rates can be developed.

\section{Authors' affiliations}

A Ogunyomade, D P S O'Brart, J Marshall, Department of

Ophthalmology, St Thomas's Hospital, London, UK

M Nagar, F Howes, Clayton Eye Centre, Wakefield, West Yorkshire, UK

This study was supported by provision of A Selectra 7000 laser by Lumenis (Coherent Medical Group, Palo Alto, CA, USA).

The authors have no proprietary interest in the Selectra 7000 laser.

Correspondence to: Mr David P S O'Brart, MD, FRCOphth, FRCS, Department of Ophthalmology, St Thomas's Hospital, Lambeth Palace Road, London SE1 7EH, UK; davidobrart@aol.com

Accepted for publication 1 June 2005

\section{REFERENCES}

1 Wise JB, Witter SL. Argon laser therapy for open-angle glaucoma: a pilot study. Arch Ophthalmol 1979;97:319-22.

2 The Glaucoma Laser Trial Research Group. The Glaucoma Laser Trial (GLT) and Glaucoma Laser Trial Follow Up Study: 7. Results. Am J Ophthalmol 1995; 120:718-31.

3 Van der Zypen E, Fankhauser F. Ultrastructural changes of the trabecular meshwork of the monkey (Macaca speciosa) following irradiation with argon laser light. Graefes Arch Clin Exp Ophthalmol, 19984, 221:249-61.

4 Melamed S, Pei J, Epstein DL. Short-term effects of argon laser trabeculoplasty in Monkeys. Arch Ophthalmol 1985; 103:1546-52.

5 Alexander RA, Grierson I. Morphological effects of argon laser trabeculoplasty upon the glaucomatous human meshwork. Eye 1989:3:719-26.

6 Latina M, Park C. Selective targeting of trabecular meshwork cells: in vitro studies of pulse and continuous laser interactions. Exp Eye Res 1995:60:359-72.

7 Latina MA, Sibayan SA, Shin DH, et al. Q-switched 532-nm Nd:YAG laser trabeculoplasty (selective laser trabeculoplasty). Ophthalmology 1998; 105:2082-90

8 Gracner T, Pahor D, Gracner B. Efficacy of selective laser trabeculoplasty in the treatment of primary open angle glaucoma. Klin Monatsbl Augenheilkd 2003;220:848-52.

9 Melamed S, Ben Simon GL, Levkovitch-Verbin H. Selective laser trabeculoplasty as primary treatment for open-angle glaucoma: a prospective, non-randomized pilot study. Arch Ophthalmol 2003;121:957-60.

10 Kano K, Kuwayama Y, Mizoue S, et al. Clinical results of selective laser trabeculoplasty. Nippon Ganka Gakkai Zasshi 1999;103:612-16.

11 Crenkel B. One-year follow-up of selective laser trabeculoplasty in open angle glaucoma. Ophthalmologica 2004;218:20-5.

12 Damii KF, Shah KC, Rock WJ, et al. Selective laser trabeculoplasty $v$ argon laser trabeculoplasty: a prospective randomized trial. $\mathrm{Br} J$ Ophthalmol 1999;83:718-22.

13 Popiela G, Muzyka M, Szelepin L, et al. Use of YAG-Selecta laser and argon laser in the treatment of open angle glaucoma. Klin Oczna 2000; 102: 129-33. 
14 Kramer TR, Noecker RJ. Comparison of the morphological changes after selective laser trabeculoplasty and argon laser trabeculoplasty in human eye bank eyes. Ophthalmology 2001;108:773-9.

15 Crenkel B, Hvala A, Drnovsek-Olup B, et al. Acute ultrastructural changes of the trabecular meshwork after selective laser trabeculoplasty and low power argon laser trabeculoplasty. Lasers Surg Med 2003;33:204-8.

16 The Glaucoma Laser Trial Research Group. The Glaucoma Laser Trial (GLT). 2. Results of argon laser trabeculoplasty versus topical medicines. Ophthalmology 1990;97:1403-13.
17 Chen E, Golchin S, Blomdahl S. A comparison between 90 degrees and 180 degrees selective laser trabeculoplasty. J Glaucoma 2004;13:62-5.

18 Gracner T. Intraocular pressure response of capsular glaucoma and primary open-angle glaucoma to selective $\mathrm{Nd}$ :YAG laser trabeculoplasty: a prospective, comparative clinical trial. Eur J Ophthalmol 2002; 12:287-92.

19 Ederer F, Gaasterland DA, Dally LG, et al. The Advanced Glaucoma Intervention Study (AGIS): 13. Comparison of treatment outcomes within race: 10-year results, Ophthalmology 2004;111:651-64.

\section{bmjupdates+}

bmiupdates+ is a unique and free alerting service, designed to keep you up to date with the medical literature that is truly important to your practice.

bmjupdates+ will alert you to important new research and will provide you with the best new evidence concerning important advances in health care, tailored to your medical interests and time demands.

Where does the information come from?

bmjupdates+applies an expert critical appraisal filter to over 100 top medical journals A panel of over 2000 physicians find the few 'must read' studies for each area of clinical interest

Sign up to receive your tailored email alerts, searching access and more...

www.bmjupdates.com 